Editorial: Hospital Metropolitano

ISSN (impreso) 1390-2989 - ISSN (electrónico) 2737-6303

Edición: Vol. 28 No 2 (2020) abril-junio

DOI: https://doi.org/10.47464/MetroCiencia/vol28/2/2020/19-24

URL: https://revistametrociencia.com.ec/index.php/revista/article/view/72

Pág: 19-24

\title{
Síndrome de Bouveret: Presentación de Caso Clínico
}

\section{Bouveret Syndrome: Clinical Case Presentation}

\section{Byron Leonel Saraguro Ramírez MD 1D; Mayra Alexandra Chiguano Chalaco MD ${ }^{2}$; Christian Ricardo Mendoza Marín MD ${ }^{3}$; Leslie Mariam Paredes Blacio MD ${ }^{4}$; Christian Mauricio Martínez Martínez ${ }^{4}$}

\author{
Centro Clínico Quirúrgico Ambulatorio Hospital del día IESS Sangolquí - Médico Neumólogo Residente Servicio de Emergencia, Quito, Ecuador \\ Centro Clínico Quirúrgico Ambulatorio Hospital del día IESS Sangolquí - Médico Radiólogo; Quito, Ecuador ${ }^{2}$ \\ Centro Clínico Quirúrgico Ambulatorio Hospital del día IESS Sangolqui - Médico Residente Servicio de Emergencia; Quito, Ecuador ${ }^{3}$ \\ Universidad Central del Ecuador - Interno Rotativo de Medicina; Quito, Ecuador ${ }^{4}$
}

Recibido: 10/02/2020 Aceptado: 15/03/2020 Públicado: 01/04/2020

\section{RESUMEN}

Introducción: El íleo biliar es una patología poco frecuente caracterizada por obstrucción mecánica intestinal debido a la impactación de un cálculo biliar dentro del tracto gastrointestinal, caracterizado por la tríada de Rigler, se observa en pacientes de edad avanzada con antecedentes de colelitiasis o colecistitis, por la existencia de una fístula bilioentérica. Se asocia a alta morbilidad y mortalidad. Sus síntomas y signos en su mayoría son inespecíficos y su diagnóstico se basa en un enfoque radiológico. Si bien la cirugía abierta ha sido el pilar del tratamiento, recientemente se han empleado otros enfoques, incluyendo cirugía laparoscópica y litotripsia. Caso clínico: Paciente femenino de 100 años de edad con cuadro de dolor abdominal, náusea y vómito de varios días de evolución. El diagnóstico fue realizado por tomografía computarizada. Conclusión: El íleo biliar es una entidad clínica de difícil diagnóstico. El uso de técnicas de imagen puede mejorar la precisión diagnóstica y la toma de recursos terapéuticos.

Palabras claves: Obstrucción Intestinal; Cálculos Biliares; Condiciones Patológicas, Signos y Síntomas; Tomografía Computarizada por Rayos X; Litotricia; Laparoscopía.

\section{ABSTRACT}

Introduction: Gallstone ileus is a rare pathology characterized by mechanical intestinal obstruction due to the impaction of a gallstone inside the gastrointestinal tract, characterized by the Rigler triad, it is observed in elderly patients with a history of cholelithiasis or cholecystitis, due to the existence of a bilioenteric fistula. It is associated with high morbidity and mortality. Its symptoms and signs are mostly nonspecific and its diagnosis is based on a radiological approach. Although open surgery has been the mainstay of treatment, other approaches have recently been used, including laparoscopic surgery and lithotripsy. Clinical case: 100 year-old female patient, with abdominal pain, nausea and vomiting that lasted several days of evolution. The diagnosis was made by computed tomography. Conclusion: Gallstone ileus is a clinical entity difficult to diagnose. The use of imaging techniques can improve the diagnostic accuracy and the taking of therapeutic resources.

Keywords: Obstrucción Intestinal; Cálculos Biliares; Condiciones Patológicas, Signos y Síntomas; Tomografía Computarizada por Rayos X; Litotricia; Laparoscopía.

Byron Leonel Saraguro Ramírez: Mayra Alexandra Chiguano Chalaco: Christian Ricardo Mendoza Marín:

Leslie Mariam Paredes Blacio: Christian Mauricio Martínez Martínez:

\section{IDs Orcid}

https://orcid.org/0000-0003-4271-3909 https://orcid.org/0000-0002-9392-3909 https://orcid.org/0000-0001-5150-6253 https://orcid.org/0000-0002-4261-951X https://orcid.org/0000-0002-2502-2455

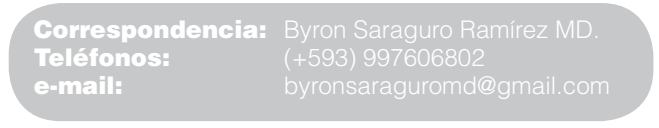




\section{INTRODUCCIÓN}

El íleo biliar, causa poco frecuente de obstrucción intestinal mecánica ${ }^{1}$, es producido por la impactación de uno o múltiples cálculos biliares grandes que se alojan en el interior del tracto gastrointestinal ${ }^{2}$, y probablemente es secundario a complicaciones de colelitiasis preexistente ${ }^{3-16}$. Bartholin la describió por primera vez en 1654 al descubrir una fístula colecistoentérica, y, en 1941 se establecieron sus signos radiológicos por Rigler, Borman y Noble, denominados tríada de Rigler consistentes en oclusión intestinal, cálculo biliar en alguna porción del intestino y neumobilia Representa del $1 \%$ al $4 \%$ de todas las obstrucciones mecánicas que se presentan con clínica inespecífica ${ }^{4,5}$.

Tiene mayor prevalencia en pacientes de edad avanzada, motivo por el cual es considerada una patología geriátrica ${ }^{9,17}$, asociada a mayor morbimortalidad $^{7}$, estimada del $8 \%$ al $30 \%{ }^{11}$ (promedio 18\%) ) $^{8,15}$, por la presencia de comorbilidades preexistentes como hipertensión, diabetes, insuficiencia cardiaca, enfermedad pulmonar crónica, anemia ${ }^{9}$, y al retraso en el diagnóstico o diagnóstico erróneo ${ }^{7}$. Es mayor en mujeres, presentándose entre el $72 \%$ al $90 \%$ y en una proporción mujer a hombre de 3,5 - 3,6:19, edad mayor a 65 años ${ }^{2}$, (promedio de presentación a los 74 años) $)^{8}$ y entre el 0,3-0,5\% de pacientes con colelitiasis9, se estima 30-35 casos por cada millón de hospitalizaciones².

El cálculo biliar usualmente causa obstrucción de la parte distal del intestino delgado donde su luz es más estrecha ${ }^{6,11}, y$, raramente causa obstrucción del duodeno ${ }^{6}$, las zonas más frecuentes de alojamiento son el íleo y la válvula íleocecal en el 60\% de los casos $^{20}$, yeyuno $16 \%$, estómago $15 \%$ y colon $2-8 \%{ }^{8}$.

Este síndrome es precedido por episodios recurrentes de colecistitis aguda ${ }^{9}$, que conduce al incremento de la presión intraluminal de la vesícula biliar secundaria a obstrucción, produciendo isquemia local y necrosis, permitiendo el paso del cálculo a través de la pared inflamada o gangrenosa ${ }^{17}$ de la vesícula biliar e ingreso a los intestinos 6 a través de una fístula bilioentérica secundaria. El 85\% de casos reportan que la fístula bilioduodenal es la más frecuente debido a su proximidad ${ }^{9}$, otros tipos de fístulas incluyen hepatoduodenales, colédocoduodenales, colecistogástricas, colecistoyeyunal, colecistoileal, y colecistocolónica ${ }^{2,8,9}$. Para lograrlo, los cálculos impactados suelen ser mayores a 2-2,5 cm de diámetro ${ }^{11}$, los cálculos de menor dimensión pasan a través del lumen intestinal y rara vez causan obstrucción?

Existen dos subtipos de íleo biliar: coleo biliar y síndrome de Bouveret.

Coleo biliar es una obstrucción mecánica del intesti- no grueso que involucra el colon transverso con pocos casos notificados en la literatura, representando el $4 \%$ de todos los casos de íleo biliar que equivale entre 12 y 15 por cada 100000 personas de todos los pacientes con enfermedad de cálculos biliares ${ }^{12}$.

El síndrome de Bouveret se presenta cuando un cálculo se aloja en el duodeno causando obstrucción a la salida gástrica ${ }^{8}$, representa del $1 \%$ al $3 \%$ de todos los casos de íleo biliar, con más prevalencia en mujeres, varias teorías indican que sea probablemente secundario al efecto colestásico de los estrógenos ${ }^{6}$. Fue reportado por Leon Bouveret en 1896 en la "Revue Medicale" presentando dos casos de obstrucción de la salida gástrica por cálculos biliares ${ }^{6}$.

\section{PRESENTACIÓN Y DIAGNÓSTICO CLÍNICO}

Los síntomas y signos clínicos son inespecíficos y dependen del sitio de la impactación del cálculo biliar? $^{7}$. Los síntomas obstructivos son intermitentes por lo que la demora diagnóstica es característica de este síndrome ${ }^{15,20}$. Pueden ser precedidos por historia de síntomas tales como dolor en cuadrante superior derecho y/o dolor abdominal generalizado tipo cólico debido a obstrucción intestinal, pérdida de peso, anorexia, saciedad temprana, náuseas, vómito que puede ser bilioso (por obstrucción del intestino delgado), o fecaloide (por obstrucción del intestino grueso) $)^{9}$, distensión abdominal y constipación que son variables, signos de hemorragia digestiva alta tales como hematemesis o melena en probable relación a la erosión de la arteria gastroduodenal. La perforación intestinal con o sin peritonitis es extremadamente rara al igual que la apendicitis gangrenosa por un cálculo impactado en la base del apéndice.

El examen físico no es específico, pueden presentar signos de deshidratación y distensión abdominal. Fiebre y signos de peritonitis pueden estar presentes si existe perforación de la pared intestinal. El examen puede ser normal si no hay obstrucción?.

Las anormalidades bioquímicas no son específicas. Pueden incluir leucocitosis, disbalance electrolítico debido a deshidratación y elevación de niveles de aminotransferasas ${ }^{17}$.

\section{Diagnóstico por Imagen}

Actualmente se han presentado más casos de íleo biliar, probablemente como resultado de un alto índice de orientación diagnóstica y la mejoría de las técnicas de diagnóstico por imagen ${ }^{7}$.

\section{Rayos X de abdomen}

Por lo general, la visualización de un cálculo biliar es complicada con rayos $X$. Su sensibilidad se sitúa entre el $40-70 \%$ y su valor predictivo positivo es del $80 \%{ }^{8}$. Los signos radiográficos son obstrucción in- 
testinal parcial o completa, neumobilia o material de contraste en el árbol biliar, cálculo aberrante o cambio de posición del cálculo al realizar radiografías seriadas. La presencia de dos de los tres primeros signos se considera patognomónico y se ha encontrado en el $20 \%$ al $50 \%$ de los casos ${ }^{9}$.

\section{Ecografía abdominal}

Es una herramienta de bajo costo, no invasiva y de elección para detección de cálculos biliares con una eficacia superior al 95\%. Con un ecografista experimentado se podría demostrar los signos de la tríada de Rigler. Además, en combinación con Rx abdominal la sensibilidad de la ecografía puede incrementarse hasta en un $74 \%{ }^{8,9}$.

\section{Tomografía computarizada}

Permite observar signos de obstrucción del intestino delgado, cálculos biliares ectópicos, anormalidades de la vesícula biliar (como presencia de aire, niveles hidroaéreos, o líquido acumulado en el interior de una pared con irregularidades) y la fistula biliodigestiva9. El estudio contrastado tiene una sensibilidad superior al 93\%, especificidad de $100 \%$ y exactitud de $99 \%{ }^{9}$. Permite además definir el nivel de la obstrucción intestinal, el tamaño y estructura del cálculo ectópico sin necesidad de administración de contraste oral8.

La TC es la modalidad diagnóstica más precisa, y constituye la mejor técnica radiológica en pacientes de emergencia por su rápida interpretación y mejor resolución ${ }^{17}$, pero requiere la validación de criterios diagnósticos. Puede incrementar la detección de cálculos aún no impactados ${ }^{11}$. Por esta razón, es considerada el gold standard ${ }^{20}$, contribuyendo a la toma de decisiones sobre el manejo del paciente sea este conservador o quirúrgico ${ }^{11,17}$

\section{Resonancia magnética}

Es considerado el estudio gold standard para la visualización del árbol biliar. La sensibilidad de la colangiopancreatografía magnética en el diagnóstico de cálculos biliares es del 97,7\% y provee detalles anatómicos del árbol biliar8, siendo útil en casos seleccionados donde el diagnóstico no es claro después del estudio tomográfico.

\section{Esofagogastroduodenoscopía}

Permite la visualización directa de una masa de consistencia dura, convexa, lisa y no friable que son características de un cálculo9 .

\section{TRATAMIENTO}

El tratamiento quirúrgico óptimo está aún sujeto a debate ${ }^{19,20}$. Hay reportes en la literatura de resolución espontánea de íleo biliar por salida de los cálculos por el recto ${ }^{11}$, y, menos común si el cálculo está en el estómago la migración proximal puede ocurrir y ser vomitado ${ }^{11}$, sin embargo, en la mayoría de pacientes el manejo quirúrgico es generalmente requerido. En pacientes ancianos y con múltiples comorbilidades existe un alto riesgo de morbilidad y mortalidad y los esfuerzos a menudo se dirigen a un manejo menos invasivo ${ }^{8}$.

No hay consensos sobre el procedimiento quirúrgico indicado. Hay diferentes opiniones sobre el tratamiento quirúrgico del íleo biliar que incluyen enterolitotomía simple, enterolitotomía más colecistectomía y cierre de fístula y enterolitotomía en una etapa y colecistectomía posterior. La enterolitotomía simple ha sido el procedimiento quirúrgico más desarrollado para aliviar la obstrucción aguda y ha sido defendida debido a su menor morbilidad y mortalidad. Se cree que la reparación de la fístula no tiene ningún beneficio adicional y podría ser potencialmente perjudicial en este grupo etáreo ${ }^{7}$, siendo asociada con complicaciones posquirúrgicas ${ }^{14,20}$.

La resección intestinal es considerada ante la presencia de isquemia, perforación o estenosis subyacente.

Muchos pacientes con íleo biliar son ancianos, en mal estado general, con varias comorbilidades, y tienen un diagnóstico tardío, que conduce a shock, deshidratación, sepsis, o peritonitis por lo que la enterolitotomía simple es el procedimiento más seguro para ellos.

Se han descrito complicaciones posquirúrgicas tales como insuficiencia renal aguda, infección del tracto urinario, íleo, fuga anastomótica, absceso intraabdominal, fístula entérica e infección de herida.

Se considera que el riesgo de recurrencia de íleo biliar varía del 2 al 5\%

\section{Endoscopía}

La primera extracción endoscópica satisfactoria fue descrita en 1985 por Bedogní.

Los cálculos biliares que causan obstrucción gastroduodenal o colónica pueden ser susceptibles de detección endoscópica y en ciertos casos de extracción. La visualización endoscópica de cálculos biliares detectados radiológicamente en el duodeno ha sido reportada, conduciendo al tratamiento quirúrgico definitivo. La tasa inicial de éxito de manejo endoscópico fue menor del 10\%. Posterior a litotripsia mecánica endoscópica, la litotripsia electrohidraúlica, por ondas de choque extracorpórea y endoscópica láser han sido usadas solas o en combinación para el manejo de cálculos biliares, sin embargo, factores como la obesidad y la interposición del intestino distendido pueden ser limitantes, además de 
la localización del cálculo fuera del alcance endoscópico ${ }^{9}$. Estos métodos endoscópicos deberían ser considerados en pacientes de edad avanzada y de alto riesgo antes de las opciones quirúrgicas en los pacientes con síndrome de Bouveret ${ }^{6}$, sin embargo, una potencial complicación del tratamiento endoscópico es la posibilidad de impactación distal de los fragmentos de los cálculos biliares.

\section{Caso clínico}

Se valoró una paciente mujer de 100 años de edad con antecedentes de hernia de pared abdominal gigante y colelitiasis. Acude con cuadro de dolor abdominal, náusea, vómito con restos hemáticos y anorexia. Presentó además una semana previa a su ingreso hematemesis que fue considerada como sangrado digestivo alto sin tratamiento específico. Al examen físico TA 80/60. FC $104, T 36,8^{\circ} \mathrm{C}$, deshidratada, dolor a la palpación en epigastrio. Los estudios de laboratorio demostraron leucocitosis

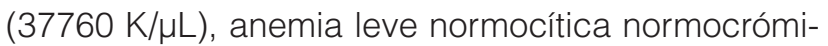
ca (Hb 10,4, Hcto 28,5\%, VCM 83,8 fL, MCHC 36 $\mathrm{g} / \mathrm{dL}$ ), trastorno hidroelectrolítico (potasio sérico 2,7 $\mathrm{mmol} / \mathrm{L}$ ), elevación de transaminasas (AST $900 \mathrm{U} / \mathrm{L}$, ALT $354 \mathrm{U} / \mathrm{L}$ ). El estudio tomográfico simple de abdomen y pelvis demostró hígado con densidad homogénea sin lesiones ocupativas evidentes, neumobilia, vesícula biliar con cálculos en su interior, gas intraluminal hacia el fondo. En la segunda porción del duodeno lesión hiperdensa circunscrita de $22 \mathrm{x}$ $14 \mathrm{~mm}$ con caracteres de cálculo biliar.

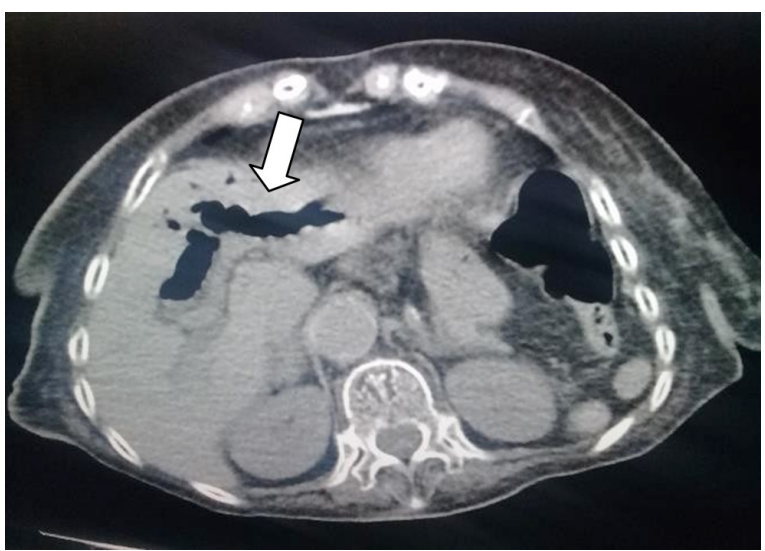

Figura 1. TC simple de abdomen corte axial: neumobilia a predominio de conducto hepático izquierdo.

Fuente: Los autores

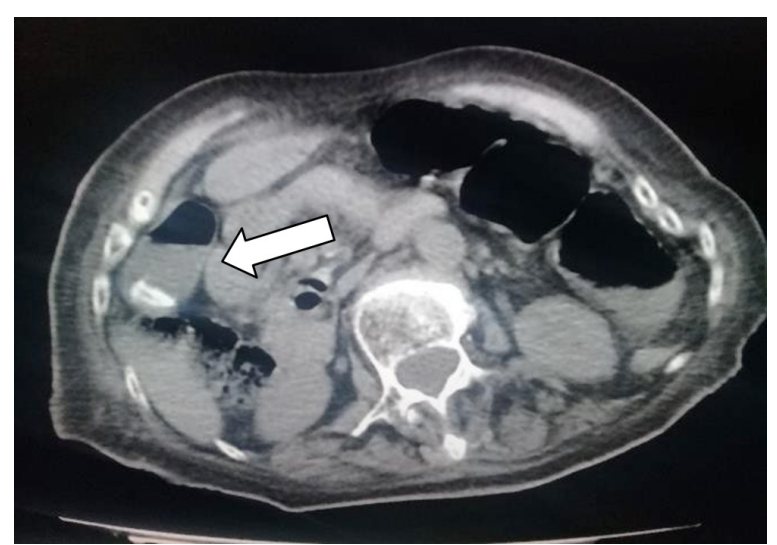

Figura 2. TC simple de abdomen corte axial: vesícula biliar con cálculos en su interior y gas hacia el fondo, no cambios en la densidad perivesicular.

Fuente: Los autores

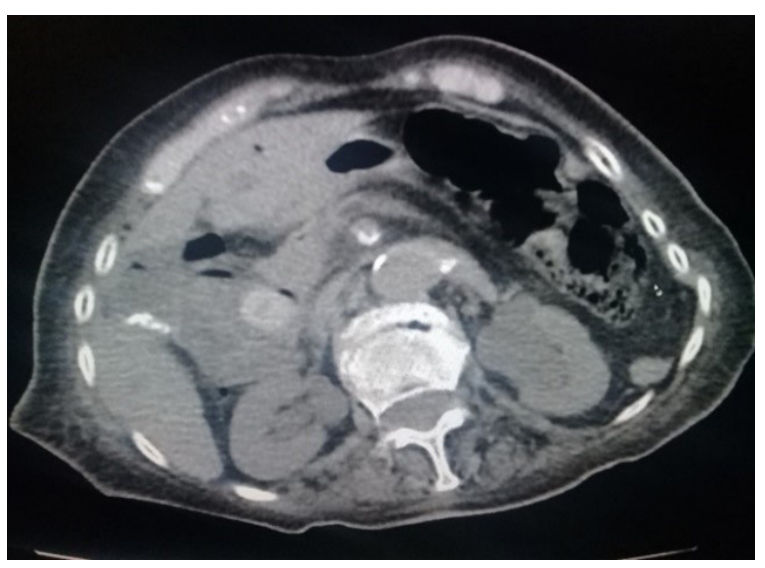

Figura 3. TC simple de abdomen corte axial: lesión hiperdensa de 22 × 14 mm circunscrita, localizada en la segunda porción del duodeno.

Fuente: Los autores

La paciente permaneció hospitalizada, se suministró cristaloides por vía parenteral para reposición hidroelectrolítica, antibioticoterapia intravenosa (ampicilina + IBL). Al no evidenciarse cuadro obstructivo intestinal se descartó la realización de procedimiento endoscópico o quirúrgico y se decidió control ambulatorio por consulta externa egresando en buenas condiciones generales.

\section{DISCusıón}

La literatura sobre el íleo biliar es retrospectiva y está constituida por el análisis de números pequeños de pacientes acumulados durante muchos años como lo describieron Ravikumar et al, constituyendo una causa poco común de obstrucción intestinal, correspondientes al 1-4\% de las obstrucciones intestinales de tipo mecánico.

La paciente descrita es de sexo femenino, dato que se correlaciona con la mayoría de la bibliografía dis- 
ponible, la cual nos indica que en comparación con los casos presentados en hombres existe una diferencia de 3,5:1 hasta 3,6:1. A pesar de esta diferencia, se han publicado varios casos de íleo biliar en pacientes de sexo masculino, uno de ellos descrito por Salamea et al.

La edad de la paciente, sobrepasó el promedio descrito de 74 años, un caso similar de un paciente de edad avanzada fue publicado por Abich et al ${ }^{17}$, que fue de 94 años.

La paciente cursó con un cuadro clínico de dolor abdominal, vómito y deshidratación tal como lo describen Murphy et al ${ }^{10}$ y García Marín et al ${ }^{13}$. Además presentó anorexia, descrito por Gadaputi et al, hematemesis reportada por Nuño-Guzmán et al ${ }^{9}$, Abich et al, y Gaduputi et al, que puede presentarse hasta en el 15\% de casos. Para el diagnóstico es útil la radiografía de abdomen cuya sensibilidad varía del 40\% al $70 \%$, sin embargo, la tomografía computarizada abdominal puede tener una exactitud diagnóstica del 99\%, siendo el método de imagen gold standard utilizado para nuestro diagnóstico. Además, la evaluación correcta del tamaño es importante porque los cálculos menores a $2 \mathrm{~cm}$ pueden ser inocuos tal como lo reportan Lassandro et al. Los estudios de laboratorio demostraron leucocitosis, disbalance electrolítico debido a deshidratación y elevación de niveles de aminotransferasas tal como lo describieron en sus reportes de casos en Abich et al, Toh et al y Besselink et $\mathrm{al}^{17-19}$.

El tratamiento definitivo es quirúrgico con el objetivo de resolver el cuadro obstructivo, y posteriormente la colecistectomía y la resolución de la fístula. En nuestro caso, al descartarse un proceso obstructivo gastrointestinal, y por la edad de la paciente, junto con la adecuada respuesta al tratamiento clínico no se determinó la resolución quirúrgica como estrategia terapéutica, tal como lo describen García Marín et $\mathrm{al}^{13}$, que de 13 pacientes sometidos a cirugía, uno fue rechazado por el equipo quirúrgico y de anestesiólogos debido al estado previo de salud (edad avanzada y dependencia de las actividades de la vida diaria). Además como lo describe Gaduputi et al, las indicaciones para cirugía abierta en síndrome de Bouveret son: cálculos mayores a 2,5 cm, cálculos residuales en vesícula biliar, múltiples cálculos en la luz intestinal, sepsis, perforación, estenosis y fallo endoscópico, que no manifestó la paciente. Nuño-Guzmán et al $^{9}$ reportaron el caso de una paciente de 91 años que no era apta para cirugía y después de la localización del cálculo biliar en la parte superior del yeyuno este fue manejado con litotripsia mecánica endoscópica.

Entre los criterios para no resolver quirúrgicamente un íleo biliar se encuentran mal estado clínico del paciente que incluye la limitación de realizar sus acti- vidades cotidianas y edad avanzada en el momento de la atención de emergencia.

Por esta razón, Kirchmayr et al21, consideran cuatro causas principales que podrían estar en relación a cursos letales: primero, es una enfermedad que se presenta en ancianos, segundo, la presencia de enfermedades concomitantes, tercero, debido a síntomas poco comunes su diagnóstico es difícil informándose el inicio de los síntomas al ingreso hospitalario y cuarto, la recuperación posoperatoria se ve obstaculizada por complicaciones como neumonía o falla cardiaca. Besselink et $\mathrm{al}^{19}$, en su reporte de caso de una paciente de 78 años demostraron como complicaciones posquirúrgicas dehiscencia de la anastomosis que requirió laparotomías reiteradas con un período de hospitalización del paciente de 24 semanas.

Lassandro et $\mathrm{al}^{11}$, reportaron 5 casos valorados en TC con presencia de neumobilia y cálculos ectópicos intestinales sin estar asociados a hallazgos de íleo mecánico lo que sugiere que una mayor precisión diagnóstica evitaría la cirugía innecesaria en pacientes cuyos cálculos pasan de manera espontánea.

\section{CONTRIBUCIÓN DE LOS AUTORES}

Byron Leonel Saraguro Ramírez: Concepción y diseño del trabajo; recolección y obtención de resultados; análisis e interpretación de datos; redacción del manuscrito.

Mayra Alexandra Chiguano Chalaco: Concepción y diseño del trabajo; recolección y obtención de resultados; análisis e interpretación de datos; redacción del manuscrito.

Christian Ricardo Mendoza Marín: Concepción y diseño del trabajo; recolección y obtención de resultados; análisis e interpretación de datos; redacción del manuscrito.

Leslie Mariam Paredes Blacio: Concepción y diseño del trabajo; recolección y obtención de resultados; análisis e interpretación de datos; redacción del manuscrito.

Christian Mauricio Martínez Martínez: Concepción y diseño del trabajo; recolección y obtención de resultados; análisis e interpretación de datos; redacción del manuscrito.

\section{CONFLICTO DE INTERÉS}

Los autores declararon no tener ningún conflicto de interés personal, financiero, intelectual, económico y de interés corporativo con el Hospital Metropolitano y los miembros de la revista MetroCiencia. 


\section{REFERENCIAS BIBLIOGRÁFICAS}

1. Ramírez m, Villanueva E, Zubieta G. Tríada de Rigler en íleo biliar. Una forma poco común de obstrucción intestinal. Revista de Gastroenterología de México 2016;81(2):103-104. https://doi.org/10.1016/j.rgmx.2015.09.002

2. Ploneda C, Gallo M, Rinchon C, Navarro E, Bautista C, de la Cerda L, Rea L, López C. El íleo biliar: una revisión de literatura médica. Revista de Gastroenterología de México 2017;82(3):248-254. https://doi.org/10.1016/j. rgmx.2016.07.006

3. Marenco B, López J, Tallón L, López J, Oliva F. ĺleo biliar colónico: una rara causa de obstrucción intestinal. Cirugía y Cirujanos 2017;85(5):440443. https://doi.org/10.1016/j.circir.2016.05.016

4. Serna J, Gutiérrez E, Juárez J, Castañeda C. Íleo biliar: Hallazgos tomográficos de la tríada de Rigler. Rev Chil Cir 2018;70(4):311-312. Disponible en: https://scielo.conicyt.cl/pdf/rchcir/v70n4/0718-4026-rchcir-70-04-0311. pdf

5. Salamea J, Salamea P. Íleo biliar, Reporte de caso clínico. Rev Fac Cienc Méd Univ Cuenca 2017;35(1):85-89. Disponible en: https://pesquisa.bvsalud.org/portal/resource/pt/biblio-999149

6. Gaduputi V, Tariq H, Rahnemai A, Dev A, Farkas D. Gallstone ileus with multiple stones: Where Rigler triad meets Bouveret's syndrome. World J Gastrointest Surg 2015;7(12):394-397. https://doi.org/10.4240/wjgs. v7.i12.394

7. Ayantunde A, Agrawal A. Gallstone lleus: Diagnosis and Management. World J Surg. 2007;31:1292-1297. https://doi.org/10.1007/s00268-0079011-9

8. Chang L, Chang M, Chang H, Chang A, Chang F. Clinical and radiologica diagnosis of gallstone ileus: a mini review. Emerg Radiol 2017. https://doi org/10.1007/s10140-017-1568-5

9. Nuño-Guzmán CM, Marín-Contreras ME, Figueroa-Sánchez M, Corona JL. Gallstone ileus, clinical presentation, diagnostic and treatment approach. World J Gastrointest Surg. 2016 Jan 27;8(1):65-76. https://doi. org/10.4240/wjgs.v8.i1.65

10. Murphy K, Kearney D, Mc Laughlin P, Maher M. Complete Radiological Findings in Gallstone lleus. J Neurogastroenterol Motil 2012;18(4):448-449. https://doi.org/ 10.5056/jnm.2012.18.4.448
11. Lassandro F, Romano S, Ragozzino A, Rossi G, Valente T, Ferrara I, Romano L, Grassi R. Role of Helical CT in Diagnosis of Gallstone Ileus and Related Conditions. AJR 2005;185:1159-1165. Disponible en: https://www. ajronline.org/doi/full/10.2214/AJR.04.1371

12. Farkas N, Kaur V, Shanmuganandan A, Black J, Redon Ch. A systematic review of gallstone sigmoid ileus management. Annals of Medicine and Surgery 2018;32-39. https://doi.org/10.1016/j.amsu.2018.01.004

13. García-Marín A, Pérez-López M, Pérez-Bru S, Compañ-Rosique A. Gallstone ileus, an uncommon cause of bowel obstruction. Rev Gastroenterol México (English Ed. 2014 Jul 1;79(3):211-3. https://doi.org/10.1016/j.rgmxen.2014.10.007

14. Ravikumar R, Graham J. The operative management of gallstone ileus. Ann R Coll Surg Eng 2010;92:279-281. https://doi.org/10.1308/00358841 OX12664192076377

15. De Monti M, Cestaro G, Alkayyali S, Galafassi J. Gallstone ileus: A possible cause of bowel obstruction in the elderly population. Internationa Journal of Surgery Case Reports 2018;18-20. https://doi.org/10.1016/j.ijscr.2018.01.010

16. Vitale J, Boni L, Brumana N. Biliary ileus. Lancet 2012;380:366. Disponible en: https://www.thelancet.com/pdfs/journals/lancet/PIIS01406736(12)60029-4.pdf

17. Abich E, Glotzer D, Murphy E. Gallstone lleus: An Unlikely Cause of Mechanical Small Bowel Obstruction. Case Rep Gastroenterol 2017;11:389395. https://doi.org/10.1159/000475749

18. Toh J, Balasuriya H, Stewart P. An Unusual Cause of Large-Bowel Obstruction: Cholecystocolonic Fistula and Gallstone Ileus. Concord Hospital Australia 2016;e108. https://dx.doi.org/10.1159/2F000456656

19. Besselink M, Kroeze J. Gallstone lleus. Mayo Clin Proc. 2005;80(5):699.

20. Athwal TS, Howard N, Belfield J, Gur U. Large bowel obstruction due to impaction of a gallstone. BMJ Case Rep. 2012;2012:bcr1120115100. https://doi.org/10.1136/bcr.11.2011.5100

21. Kirchmayr W, Mühlmann G, Zitt M, Bodner J, Weiss H, Klaus A. Gallstone ileus: Rare and still controversial. ANZ J Surg. 2005 Apr;75(4):234-8. https://doi.org/10.1111/j.1445-2197.2005.03368.x 